\title{
Controle de plantas daninhas e seletividade do herbicida tembotrione na cultura do milho
}

\author{
Weeds control and selectivity of tembotrione herbicide in the corn culture
}

\author{
Jeferson Zagonel ${ }^{1}$, Eliana Cuéllar Fernandes ${ }^{2}$
}

\begin{abstract}
Resumo - Objetivando avaliar a eficiência e a seletividade do herbicida tembotrione, isolado e complementado com atrazina no controle de plantas daninhas na cultura do milho, instalou-se um experimento na Fazenda Escola da Universidade Estadual de Ponta Grossa, no ano agrícola 2004/05. O delineamento experimental foi de blocos ao acaso com nove tratamentos e quatro repetições. Os tratamentos constaram da aplicação em pós-emergência de $0,12 \mathrm{~L} \mathrm{ha}^{-1}$ de Equip Plus (foransulfuron \& iodosulfuron methyl sodium) complementado com $2 \mathrm{~L} \mathrm{ha}^{-1}$ de Atrazinax (atrazina) e de 1,0 L ha' ${ }^{-1}$ de Hoefix (espalhante adesivo); 0,60 $\mathrm{L} \mathrm{ha}^{-1}$ de Sanson (nicosulfuron) complementado com 2,0 L ha ${ }^{-1}$ de Atrazinax; 0,30 $\mathrm{L} \mathrm{ha}^{-1}$ de Callisto (mesotrione) complementado com 3,0 L ha ${ }^{-1}$ de Primóleo (atrazina); Soberan (tembotrione) nas doses de 0,18 e 0,24 $\mathrm{L} \mathrm{ha}^{-1}$ complementado com $2 \mathrm{~L} \mathrm{ha}^{-1}$ de Atrazinax e adicionados de $1,0 \mathrm{~L} \mathrm{ha}^{-1}$ de Aureo (óleo metilado de soja) e nas doses de 0,24 e $0,30 \mathrm{~L} \mathrm{ha}^{-1}$ adicionado de $1,0 \mathrm{~L} \mathrm{ha}^{-1}$ de Aureo; testemunha capinada e testemunha sem capina. O híbrido utilizado foi DKB-215. O Soberan complementado com atrazina é eficiente no controle para Brachiaria plantaginea, Digitaria horizontalis e Bidens pilosa, com resultados similares a Callisto + Atrazinax e iguais ou superiores a Equip Plus e a Sanson + Atrazinax. Quando aplicado isolado, o Soberan mostrou igual resultado para as gramíneas, porém, para Bidens pilosa o controle foi eficiente, mas inferior a alguns tratamentos; o Soberan isolado, ou em mistura com Atrazinax, causou leves sintomas de fitotoxicidade até 14 dias após sua aplicação, tal qual a mistura de Equip Plus e de Sanson + Atrazinax.
\end{abstract}

Palavras-chave: Zea mays, plantio direto

\begin{abstract}
With the aim of evaluate the efficiency and the selectivity of the tembotrione herbicide, isolated and complemented with atrazine, in the weeds control of the corn culture, an experiment was installed at the UEPG School Farm, in the 2004/05 agricultural year. The experimental design was a complete randomized blocks with nine treatments and four repetitions. The treatments consisted of the postemergence application of Equip Plus (foransulfuron \& iodosulfuron methyl sodium) - 0,12 $\mathrm{L} \mathrm{ha}^{-1}$ complemented with Atrazinax (atrazine) - $2 \mathrm{~L} \mathrm{ha}^{-1}$ and Hoefix (adjuvant) - 1,0 L ha ${ }^{-1}$; Sanson (nicosulfuron) - 0,60 $\mathrm{L} \mathrm{ha}^{-1}$ complemented with Atrazinax - 2,0 L ha ${ }^{-1}$; Callisto (mesotrione) - 0,30 L ha ${ }^{-1}$ complemented with Primóleo (atrazine) - 3,0 L ha ; Soberan (tembotrione) - 0,18 and 0,24 $\mathrm{L} \mathrm{ha}^{-1}$ complemented with Atrazinax - $2 \mathrm{~L} \mathrm{ha}^{-1}$ and added of Aureo (metilado oil of soy) - 1,0 L ha ${ }^{-1}$; Soberan (tembotrione) - 0,24 and 0,30 $\mathrm{L} \mathrm{ha}^{-1}$
\end{abstract}

\footnotetext{
${ }^{1}$ Prof. Associado, DEFITO/UEPG, Praça Santos Andrade, nº1, Ponta Grossa, PR, 84.010-919, e-mail: jefersonzagonel@uol.com.br;

${ }_{2}$ Mestranda em Agricultura, UEPG, Rua Mathias de Albuquerque, nº577, Ponta Grossa, PR, 84.036-140, e-mail: elianacfernandes@uol.com.br.
} 
added of Aureo (metilado oil of soy) - 1,0 $\mathrm{L} \mathrm{ha}^{-1}$; with and without weeding. The used hybrid was DKB-215. Soberan complemented with Atrazinax is efficient in the control for Brachiaria plantaginea, Digitaria horizontalis e Bidens pilosa, with similar results to Callisto + Atrazinax and equal or superior results to Equip Plus and Sanson + Atrazinax. When applied isolated Soberan it showed equal result for the grassy ones, however, for Bidens pilosa the control was efficient, but inferior to some treatments; Soberan in the biggest rate and with Atrazinax after promoted light symptoms of fitotoxicity up to 14 days its application, such which the mixture of Equip Plus and Sanson + Atrazinax.

Key-words: Zea mays, no tillage.

\section{Introdução}

Os efeitos negativos causados pela mato-competição nas lavouras é bem conhecido. Esses se agravam à medida que a produtividade da cultura em questão aumenta, visto que mesmo perdas pequenas se refletem em danos na produção e redução dos lucros. No caso do milho não é difícil observar produtividades superiores a $13.000 \mathrm{~kg} \mathrm{ha}^{-1}$, o que requer um controle de todos os fatores que afetam a cultura. Nesse sentido, as plantas daninhas devem ser controladas adequadamente e na época correta para que não venham a interferir no desenvolvimento e na produtividade da cultura.

Em geral, o controle das infestantes no milho é realizado com herbicidas de pósemergência, pela flexibilidade da escolha do produto e da dose a serem aplicados para as espécies presentes, e também em função da infestação da área. Nessa modalidade de aplicação a seletividade dos herbicidas é muito importante (Vidal et al., 2002), junto com a época de aplicação dos herbicidas. Essa varia em função da espécie presente, de sua população e da época que incidem (Zagonel et al., 2000), e em geral a cultura deve ser mantida livre da presença das plantas daninhas entre 20 e 45 dias após a emergência (Blanco et al., 1978).

No entanto, quando a infestação é predominantemente de poáceas (gramíneas) e/ou para altas populações de plantas daninhas incidindo desde o início do desenvolvimento da cultura, o controle deve ser executado mais cedo (Pitelli, 1985; Zagonel et al., 1998), sendo denominado de controle em pós-inicial ou pósprecoce. Nessa época, em geral as gramíneas estão no estádio de 1 a 3 folhas até o perfilhamento, e as espécies latifoliadas estão com 2 a 4 folhas no máximo. Nesse estádio das plantas daninhas os herbicidas são mais eficientes, pois a suscetibilidade das infestantes é maior.

Várias são as opções de controle das plantas daninhas no milho, e entre os herbicidas utilizados na cultura encontram-se a atrazina, o nicosulfuron, o mesotrione e a mistura pronta de foransulfuron com iodosulfuron-metil-sodium (Zagonel, 2002; Zagonel, 2003). Outro herbicida, o tembotrione, do grupo das isoxazolidinonas, pode constituir em mais uma opção de controle desde que sua eficácia e seletividade sejam avaliados. Nesse sentido, realizou-se o presente trabalho objetivando avaliar a eficiência e a seletividade do herbicida tembotrione, isolado e complementado com atrazina no controle de plantas daninhas na cultura do milho.

\section{Material e métodos}

O experimento foi instalado na Fazenda Escola da Universidade Estadual de Ponta Grossa, no município de Ponta Grossa, PR, em um Cambisolo Háplico Tb distrófico típico, de textura argilosa, no ano agrícola 2004/05. O 
delineamento experimental utilizado foi de blocos ao acaso com nove tratamentos e quatro repetições.

O sistema de plantio utilizado foi o "plantio direto na palha", com semeadura realizada mecanicamente em fileiras espaçadas de $0,80 \mathrm{~m}$, semeando-se de 5 a 6 sementes por metro, a uma profundidade de $0,05 \mathrm{~m}$. O híbrido utilizado foi DKB-215, com semeadura efetuada em 01/10/04. A adubação consistiu da aplicação de $300 \mathrm{~kg} \mathrm{ha}^{-1}$ de adubo de fórmula 04-20-20 na semeadura e $90 \mathrm{~kg} \mathrm{ha}^{-1} \mathrm{de}$ nitrogênio (na forma de uréia), aplicado em cobertura aos 11 dias após a emergência do milho.

Os tratamentos constaram da aplicação em pós-emergência de $0,12 \mathrm{~L} \mathrm{ha}^{-1}$ de Equip Plus (foransulfuron \& iodosulfuron methyl sodium) complementado com $2 \mathrm{~L} \mathrm{ha}^{-1} \mathrm{de}$ Atrazinax (atrazina) e de $1,0 \mathrm{~L} \mathrm{ha}^{-1}$ de Hoefix (espalhante adesivo); 0,60 $\mathrm{L} \mathrm{ha}^{-1}$ de Sanson (nicosulfuron) complementado com 2,0 L ha de Atrazinax; 0,30 $\mathrm{L}$ ha $^{-1}$ de Callisto (mesotrione) complementado com 3,0 $\mathrm{L} \mathrm{ha}^{-1}$ de Primóleo (atrazine); Soberan (tembotrione) nas doses de 0,18 e $0,24 \mathrm{~L} \mathrm{ha}^{-1}$ complementado com $2 \mathrm{~L} \mathrm{ha}^{-1}$ de Atrazinax e adicionados de 1,0 $\mathrm{L} \mathrm{ha}^{-1}$ de Aureo (óleo metilado de soja) e nas doses de 0,24 e 0,30 $\mathrm{L} \mathrm{ha}^{-1}$ adicionado de 1,0 L ha $^{-1}$ de Aureo; testemunha capinada e testemunha sem capina. Os herbicidas foram aplicados através de pulverizador costal, à pressão constante de $206,85 \mathrm{kPa}$, pelo $\mathrm{CO}_{2}$ comprimido, com pontas de jato plano "leque" XR 110-02. Aplicou-se o equivalente a $200 \mathrm{~L}$ $\mathrm{ha}^{-1}$ de calda. A aplicação dos tratamentos foi realizada no milho com quatro folhas. As plantas daninhas presentes no experimento, com respectivo número de plantas por metro quadrado e estádio de desenvolvimento, avaliadas no dia da aplicação dos tratamentos, encontram-se na Tabela 1.

Tabela 1. Nomes científico e comum, número de plantas $\mathrm{m}^{-2}$ e estádio de desenvolvimento das plantas daninhas presentes no experimento com milho. UEPG. Ponta Grossa, PR. 2004/05.

\begin{tabular}{lccc}
\hline \multicolumn{1}{c}{ Nome científico } & Nome comum & ${\text { Plantas } \mathbf{~ m}^{-2}}$ & Estádio de desenvolvimento \\
\hline Brachiaria plantaginea & capim-papuã & 34 & 2 folhas a 1 perfilho \\
Digitaria horizontalis & capim-milhã & 62 & 1 folha a 1 perfilho \\
Bidens pilosa & picão-preto & 28 & 2 a 4 folhas \\
\hline
\end{tabular}

As avaliações de controle de plantas daninhas foram efetuadas visualmente aos 7 , 14, 28 e 50 dias após a aplicação dos tratamentos. A metodologia de avaliação utilizada foi a visual (Sociedade Brasileira da Ciência das Plantas Daninhas, 1995), comparando-se o controle dos herbicidas com a testemunha sem capina, onde "0\%" correspondeu a "sem controle" e "100\%" a "controle total". As avaliações de fitotoxicidade foram efetuadas aos 7, 14 e 28 DAA, utilizando-se como base os conceitos da
Sociedade Brasileira da Ciência das Plantas Daninhas (1995).

A colheita foi efetuada no dia 15/04/2005 utilizando-se as plantas da área útil das parcelas. Os dados foram submetidos à análise da variância pelo teste $\mathrm{F}$ e as diferenças entre as médias comparadas pelo teste de Tukey $(\mathrm{p}<0,05)$. Na análise das avaliações de controle foram comparados somente os resultados dos tratamentos herbicidas, isolando-se as testemunhas. 


\section{Resultados e discussão}

O clima no decorrer do experimento foi adequado ao desenvolvimento do milho e das plantas daninhas, que se encontravam em plena atividade metabólica na época da aplicação dos tratamentos. Todos os herbicidas promoveram controle eficiente (igual ou superior a $80 \%$ ) para Brachiaria plantaginea em todas as avaliações (Tabela 2).

$\mathrm{Na}$ avaliação aos 7 dias após a aplicação (DAA) dos tratamentos, o controle dos tratamentos foi menor que nas demais avaliações, nas quais o potencial de controle dos mesmos foi exteriorizado. Aos 7 DAA não foram observadas diferenças substanciais no controle entre os tratamentos, o que ocorreu nas demais avaliações, em que, aos 14 DAA o Equip Plus + Atrazinax e o Sanson + Atrazinax foram de controle inferior ao Soberan (com e sem Atrazinax), tal qual ocorreu aos 50 DAA.

Aos 28 DAA o Equip Plus + Atrazinax foi de controle inferior aos demais tratamentos, e Sanson + Atrazinax e Callisto + Primóleo foram de resultados inferiores aos verificados aos tratamentos com Soberan. Para esse último produto não foram observadas diferenças entre as doses e vantagem da adição ou não de atrazina para o controle de B. plantaginea. Constantin et al. (2006) e Blanco et al. (2006) também avaliaram o controle do Soberan para B. plantaginea e observaram controle superior a $90 \%$ tanto para o Soberan isolado como em mistura com atrazina, o que mostra a efetividade desse herbicida no controle dessa espécie.

Tabela 2. Avaliação visual de controle (\%) para Brachiaria plantaginea, Digitaria horizontalis e Bidens pilosa na cultura do milho. Fazenda Escola (UEPG). Ponta Grossa, PR. 2004/05.

Controle (\%) para Brachiaria plantaginea

\begin{tabular}{|c|c|c|c|c|c|}
\hline Nome comercial & Dose $\left(p c \text { ha }^{-1}\right)^{1}$ & $7 \mathbf{D A A}^{2}$ & 14 DAA & 28 DAA & 50 DAA \\
\hline 1. Equip Plus + Atrazinax & $0,12 \mathrm{~L}+2,00 \mathrm{~L}$ & $80,0 \mathrm{a}$ & $90,0 \quad c$ & $85,0 \quad \mathrm{c}$ & $83,8 \mathrm{~b}$ \\
\hline 2. Sanson + Atrazinax & $0,60 \mathrm{~L}+2,00 \mathrm{~L}$ & $80,0 \mathrm{a}$ & $91,3 \mathrm{bc}$ & $95,0 \mathrm{~b}$ & $88,3 \mathrm{~b}$ \\
\hline 3. Callisto + Primóleo & $0,30 \mathrm{~L}+3,00 \mathrm{~L}$ & $83,8 \mathrm{a}$ & $96,5 \mathrm{ab}$ & $95,8 \mathrm{~b}$ & $98,0 \mathrm{a}$ \\
\hline 4. Soberan + Atrazinax ${ }^{3}$ & $0,18 \mathrm{~L}+2,00 \mathrm{~L}$ & 85,0 a & $97,3 \mathrm{a}$ & $98,0 \mathrm{a}$ & $98,0 \mathrm{a}$ \\
\hline 5. Soberan + Atrazinax $^{3}$ & $0,24 \mathrm{~L}+2,00 \mathrm{~L}$ & $81,8 \mathrm{a}$ & $98,0 \mathrm{a}$ & $98,0 \mathrm{a}$ & $98,0 \mathrm{a}$ \\
\hline 6. Soberan ${ }^{3}$ & $0,24 \mathrm{~L}$ & 81,3 a & $98,0 \mathrm{a}$ & $98,3 \mathrm{a}$ & $98,0 \mathrm{a}$ \\
\hline 7. Soberan ${ }^{3}$ & $0,30 \mathrm{~L}$ & $81,3 \mathrm{a}$ & $98,0 \mathrm{a}$ & $98,0 \mathrm{a}$ & $98,0 \mathrm{a}$ \\
\hline \multirow[t]{2}{*}{ C.V. $(\%)^{4}$} & & 3,8 & 2,5 & 0,6 & 2,1 \\
\hline & & \multicolumn{4}{|c|}{ Controle (\%) para Digitaria horizontalis } \\
\hline 1. Equip Plus + Atrazinax & $0,12 \mathrm{~L}+2,00 \mathrm{~L}$ & $73,8 \mathrm{~b}$ & $90,0 \mathrm{c}$ & $90,0 \mathrm{~b}$ & $83,8 \mathrm{~b}$ \\
\hline 2. Sanson + Atrazinax & $0,60 \mathrm{~L}+2,00 \mathrm{~L}$ & $81,3 \mathrm{ab}$ & $91,3 \mathrm{bc}$ & $95,8 \mathrm{a}$ & $91,3 \mathrm{ab}$ \\
\hline 3. Callisto + Primóleo & $0,30 \mathrm{~L}+3,00 \mathrm{~L}$ & $85,0 \mathrm{a}$ & $96,5 \mathrm{ab}$ & $95,8 \mathrm{a}$ & $91,3 \mathrm{ab}$ \\
\hline 4. Soberan + Atrazinax $^{3}$ & $0,18 \mathrm{~L}+2,00 \mathrm{~L}$ & $85,0 \mathrm{a}$ & $97,3 \mathrm{a}$ & $92,0 \mathrm{ab}$ & $92,5 \mathrm{a}$ \\
\hline
\end{tabular}




\begin{tabular}{|c|c|c|c|c|c|}
\hline 5. Soberan + Atrazinax $^{3}$ & $0,24 \mathrm{~L}+2,00 \mathrm{~L}$ & $83,8 \mathrm{a}$ & 98,0 a & $93,8 \mathrm{ab}$ & $92,5 \mathrm{a}$ \\
\hline 6. Soberan ${ }^{3}$ & $0,24 \mathrm{~L}$ & $82,5 \mathrm{ab}$ & $98,0 \mathrm{a}$ & $96,5 \mathrm{a}$ & $96,5 \mathrm{a}$ \\
\hline 7. Soberan ${ }^{3}$ & $0,30 \mathrm{~L}$ & $83,0 \mathrm{a}$ & $98,0 \mathrm{a}$ & $95,8 \mathrm{a}$ & $96,5 \mathrm{a}$ \\
\hline \multirow[t]{2}{*}{ C.V. $(\%)^{4}$} & & 4,6 & 2,5 & 2,3 & 3,5 \\
\hline & & \multicolumn{4}{|c|}{ Controle (\%) para Bidens pilosa } \\
\hline 1. Equip Plus + Atrazinax & $0,12 \mathrm{~L}+2,00 \mathrm{~L}$ & $87,5 \mathrm{a}$ & $92,5 \mathrm{ab}$ & $91,3 \mathrm{~b}$ & $85,0 \mathrm{~b}$ \\
\hline 2. Sanson + Atrazinax & $0,60 \mathrm{~L}+2,00 \mathrm{~L}$ & $81,3 \mathrm{ab}$ & $91,3 \mathrm{bc}$ & $93,8 \mathrm{ab}$ & 93,8 a \\
\hline 3. Callisto + Primóleo & $0,30 \mathrm{~L}+3,00 \mathrm{~L}$ & 88,8 a & $95,8 \mathrm{ab}$ & $95,8 \mathrm{ab}$ & $95,0 \mathrm{a}$ \\
\hline 4. Soberan + Atrazinax ${ }^{3}$ & $0,18 \mathrm{~L}+2,00 \mathrm{~L}$ & 83,3 a & $98,0 \mathrm{a}$ & 97,3 a & $95,0 \mathrm{a}$ \\
\hline 5. Soberan + Atrazinax ${ }^{3}$ & $0,24 \mathrm{~L}+2,00 \mathrm{~L}$ & $85,0 \mathrm{a}$ & $98,0 \mathrm{a}$ & $98,0 \mathrm{a}$ & $96,5 \mathrm{a}$ \\
\hline 6. Soberan ${ }^{3}$ & $0,24 \mathrm{~L}$ & $73,8 \mathrm{~b}$ & $85,0 \mathrm{~cd}$ & $81,3 \mathrm{c}$ & $82,5 \mathrm{~b}$ \\
\hline 7. Soberan ${ }^{3}$ & $0,30 \mathrm{~L}$ & $72,5 \mathrm{~b}$ & $84,5 \mathrm{~d}$ & $81,3 \mathrm{c}$ & $82,5 \mathrm{~b}$ \\
\hline 8. Testemunha capinada & $\begin{array}{ll}--- \\
---\end{array}$ & 0,0 & 0,0 & 0,0 & 0,0 \\
\hline 9. Testemunha sem capina & ---- & 100,0 & 100,0 & 100,0 & 100,0 \\
\hline C.V. (\%) & & 4,9 & 2,9 & 2,2 & 2,8 \\
\hline
\end{tabular}

Médias seguidas da mesma letra nas colunas não diferem significativamente pelo teste de Tukey (p<5\%); ${ }^{1}$ Dose do produto comercial por hectare; ${ }^{2}$ Dias após a aplicação dos tratamentos; ${ }^{3}$ Tratamentos adicionados de $1,0 \mathrm{~L} \mathrm{ha}{ }^{-1}$ de Aureo (óleo metilado de soja); ${ }^{4}$ C.V. = coeficiente de variação.

No controle de Digitaria horizontalis (Tabela 2), aos 7 DAA somente o Equip Plus + Atrazinax não foi de controle superior a $80 \%$ e portanto, considerado eficiente. Nas demais avaliações todos os tratamentos promoveram controle eficiente da infestante em questão, mas com diferenças na eficácia.

Aos 14 DAA o Equip Plus + Atrazinax e o Sanson + Atrazinax foram de controle inferior aos tratamentos com o Soberan. Aos 28 DAA o Equip Plus + Atrazinax mostrou menor porcentagem de controle em relação a Sanson + Atrazinax, Callisto + Primóleo e Soberan isolado, e aos 50 DAA foi de controle inferior ao Soberan (com e sem Atrazinax).

Em relação ao Soberan, não foi observada vantagem da adição de atrazina e nem diferenças entre as doses, visto que em todas o controle foi eficiente e similar ao Sanson + atrazinax.

No presente trabalho as plantas de $D$. horizontalis se encontravam com uma folha a um perfilho (Tabela 1). Em uma avaliação do Soberan em plantas mais desenvolvidas, Ferreira et al. (2006) verificaram controle superior a $90 \%$ para Callisto e Soberan, o que corrobora com os resultados do presente trabalho. No entanto, para Equip Plus e Sanson o controle observado pelos autores foi muito baixo, ao contrário do observado na Tabela 2.

Os tratamentos com Soberan, em ambas as doses, foram os únicos que mostraram controle eficiente para Bidens pilosa aos 7 DAA. Nas demais avaliações, todos os tratamentos promoveram controle da infestante em questão (Tabela 2). Aos 14, 28 e 
50 DAA, nota-se que o Soberan aplicado isoladamente foi de controle eficiente, mas inferior à maioria dos tratamentos. No entanto, deve-se destacar que exceto para o Equip Plus + Atrazinax, os demais foram complementados com atrazina, que é altamente eficiente no controle de Bidens pilosa. Mesmo assim deve ser considerada a eficácia de controle do Equip Plus isolado e principalmente o excelente controle do Soberan quando complementado com atrazina, que superou o Sanson +
Atrazinax aos 14 DAA e foi similar ao Callisto + Primóleo em todas as avaliações.

Para a produtividade (Tabela 3) verificou-se valores similares entre a testemunha capinada e os tratamentos com herbicidas, que por sua vez mostraram resultados bastante superiores ao da testemunha sem capina, o que ocorreu pelo número elevado de plantas daninhas, especialmente de folhas estreitas. .

Tabela 3. Produtividade de grãos $\left(\mathrm{kg} \mathrm{ha}^{-1}\right)$ e fitotoxicidade dos tratamentos de controle de plantas daninhas aplicados no milho. Fazenda Escola - UEPG. Ponta Grossa, PR. 2004/05.

\begin{tabular}{|c|c|c|c|c|}
\hline \multirow{2}{*}{$\begin{array}{c}\text { Nome } \\
\text { Comercial }\end{array}$} & \multirow{2}{*}{$\begin{array}{c}\text { Dose } \\
\left(\mathrm{pc} \mathrm{ha}^{-1}\right)\end{array}$} & \multirow{2}{*}{$\begin{array}{l}\text { Produtividade } \\
\left(\mathrm{kg} \mathrm{ha}^{-1}\right)\end{array}$} & \multicolumn{2}{|c|}{ Fitotoxicidade $^{1}(\%)$} \\
\hline & & & 7 DAA $^{2} 14$ DAA & 28 DAA \\
\hline 1. Equip Plus + Atrazinax & $0,12 \mathrm{~L}+2,00 \mathrm{~L}$ & $8.578 \mathrm{a}$ & $\mathrm{b}$ & $\mathrm{a}$ \\
\hline 2. Sansón + Atrazinax & $0,60 \mathrm{~L}+2,00 \mathrm{~L}$ & $9.021 \mathrm{a}$ & $\mathrm{b}$ & $\mathrm{a}$ \\
\hline 3. Callisto + Primóleo & $0,30 \mathrm{~L}+3,00 \mathrm{~L}$ & $9.385 \mathrm{a}$ & $\mathrm{a}$ & a \\
\hline 4. Soberan + Atrazinax $^{3}$ & $0,18 \mathrm{~L}+2,00 \mathrm{~L}$ & $9.328 \mathrm{a}$ & $\mathrm{b}$ & $\mathrm{a}$ \\
\hline 5. Soberan + Atrazinax $^{3}$ & $0,24 \mathrm{~L}+2,00 \mathrm{~L}$ & $9.292 \mathrm{a}$ & $\mathrm{b}$ & a \\
\hline 6. Soberan ${ }^{3}$ & $0,24 \mathrm{~L}$ & $9.266 \mathrm{a}$ & $\mathrm{b}$ & $\mathrm{a}$ \\
\hline 7. Soberan ${ }^{3}$ & $0,30 \mathrm{~L}$ & $9.771 \mathrm{a}$ & $\mathrm{b}$ & $\mathrm{a}$ \\
\hline 8. Testemunha capinada & ---- & $8.880 \mathrm{a}$ & $\mathrm{a}$ & $\mathrm{a}$ \\
\hline 9. Testemunha sem capina & ---- & $2.630 \mathrm{~b}$ & $\mathrm{a}$ & $\mathrm{a}$ \\
\hline C.V. $(\%)$ & ---- & 6,0 & --- & --- \\
\hline $\begin{array}{l}\text { Conceito conforme SBCPD (199 } \\
\text { (óleo metilado de soja); }{ }^{4} \mathrm{C} . \mathrm{V} .=\mathrm{co} \\
\text { Em relação a fitotox } \\
\text { observou-se um comporta } \\
\text { entre os herbicidas. Para } \\
\text { Sanson e para Soberan (iso } \\
0,24 \text { e } 0,30 \mathrm{~L} \mathrm{ha}^{-1} \text { ) e co } \\
\text { Atrazinax (dose de } 0,24 \\
\text { observados sintomas até } \\
\text { Soberan, na dose de } \\
\text { complementado com }\end{array}$ & $\begin{array}{l};^{2} \text { Dias após a aplicas } \\
\text { ficiente de variação. } \\
\text { idade (Tabela 3), } \\
\text { hento diferencial } \\
\text { Equip Plus, o } \\
\text { ado nas doses de } \\
\text { plementado com } \\
\text { L ha }{ }^{-1} \text { ) foram } \\
4 \text { DAA. Para o } \\
0,18 \text { L ha }{ }^{-1} \text {, } \\
\text { trazinax foram }\end{array}$ & $\begin{array}{l}\text { ão dos tratamentos; } \\
\text { acentuados e nã } \\
\text { avaliação aos } 2 \\
\text { não vieram a a } \\
\text { indicando seleti } \\
\text { Para o Callisto } \\
\text { fitotoxicidade. } \\
\text { Constantin et al. } \\
\text { leve fitotoxicidi } \\
\text { clorose, mas qu }\end{array}$ & $\begin{array}{l}{ }^{3} \text { Adicionados de } 1,0 \mathrm{~L} \\
\text { ão foram mais obs } \\
8 \text { DAA. Além diss } \\
\text { fetar a produção } \\
\text { vidade dos mesmos } \\
\text { não se observou s } \\
\text { Resultados obt } \\
\text { (2006) também mo } \\
\text { ade inicial, caracte } \\
\text { le não foi mais ide } \\
\text { após a aplicação do }\end{array}$ & $\begin{array}{l}a^{-1} \text { de Aureo } \\
\text { ervados na } \\
\text { o, também } \\
\text { Tabela 2), } \\
\text { à cultura. } \\
\text { ntomas de } \\
\text { idos por } \\
\text { stram uma } \\
\text { rizada por } \\
\text { ntificada a } \\
\text { Soberan. }\end{array}$ \\
\hline
\end{tabular}
observados sintomas até 7 DAA. Deve-se destacar que os sintomas observados não foram 


\section{Conclusões}

Nesse experimento concluiu-se que o Soberan, complementado com Atrazinax, é eficiente no controle para Brachiaria plantaginea, Digitaria horizontalis e Bidens pilosa, com resultados similares a Callisto complementado com Primóleo e iguais ou superiores a Equip Plus e a Sanson complementados com Atrazinax.

Quando aplicado isolado, o Soberan controlou eficientemente as gramíneas e Bidens pilosa, mas, para essa última o controle foi inferior à maioria dos tratamentos; o Soberan promoveu leves sintomas de fitotoxicidade até 14 DAA (com e sem Atrazinax), tal qual ocorreu para Equip Plus e Sanson complementados com Atrazinax, sintomas não mais observados aos $28 \mathrm{DAA}$

\section{Referências}

BLANCO, H.G., OLIVEIRA, D.A., ARAÚJO, J.B.M. Épocas em que uma associação de mato provoca prejuízos por competição na cultura do milho. In: SEMINÁRIO BRASILEIRO DE HERBICIDAS E PLANTAS DANINHAS, 12. Fortaleza, 1978. Anais... Fortaleza: SBCPD, 1978. p.18.

BLANCO, F. M. G.; SUZUKI, M. Y.; FRANCO D. A. de S. Avaliação da eficiência do herbicida tembotrione (Soberan), aplicado de forma isolada e como componente de misturas no controle das plantas daninhas na cultura do milho. In: CONGRESSO BRASILEIRO DA CIÊNCIA DAS PLANTAS DANINHAS, 25. Brasília - DF, 2006. Anais... Brasília, SBCPD, 2006. (CD-ROM).

CONSTANTIN, J.; OLIVEIRA JR. R.S.; BLAINSKI, E.; HOMEM, L.M. Seletividade e eficácia agronômica do novo herbicida tembotrione para a cultura do milho. In: CONGRESSO BRASILEIRO DA CIÊNCIA
DAS PLANTAS DANINHAS, 25. Brasília DF, 2006. Anais... Brasília, SBCPD, 2006. (CD-ROM).

FERREIRA, L.R. et al. Eficiência do tembotrione no manejo de plantas daninhas na cultura do milho. In: CONGRESSO BRASILEIRO DA CIÊNCIA DAS PLANTAS DANINHAS, 25. Brasília - DF, 2006. Anais... Brasília, SBCPD, 2006. (CD-ROM).

PITELLI, R.A. Interferência das plantas daninhas nas culturas agrícolas. Informe Agropecuário, v.11, n.1, p.16-27, 1985.

SOCIEDADE BRASILEIRA DA CIÊNCIA DAS PLANTAS DANINHAS. Procedimentos para instalação, avaliação e análise de experimentos com herbicidas. Londrina: SBCPD, 1995. $42 \mathrm{p}$.

VIDAL, R.A.; SPADER, V.; FLECK, N.G.; MEROTTO JUNIOR, A. Dose de injúria econômica do herbicida cyanazine na cultura do milho. Revista Brasileira de Herbicidas, v.3, n.2/3, p.127-132, 2002.

ZAGONEL, J., CARVALHO, O.A., KUNZ, R.P. Interferência das plantas daninhas na produtividade e características agronômicas do milho. In: CONGRESSO NACIONAL DE MILHO E SORGO, 22. Recife - PE, 1998. Anais... Recife, ABMS, EMBRAPA, 1998. p. 220 .

ZAGONEL, J., VENANCIO, W.S., KUNZ, R.P. Efeitos de métodos e épocas de controle das plantas daninhas na cultura do milho. Planta Daninha, v.18, n.1, p.143-150, 2000.

ZAGONEL, J. Eficácia do herbicida mesotrione isolado e em mistura com atrazina no controle de plantas daninhas na cultura do milho. In: CONGRESSO BRASILEIRO DA CIÊNCIA DAS PLANTAS DANINHAS, 23. 
Gramado, 2002, Anais... Londrina: SBCPD, 2002, p. 658.

ZAGONEL, J. Eficiência comprovada.

Cultivar, v.5, n.43, p.30-32, 2003. 